Pacific Journal of Mathematics

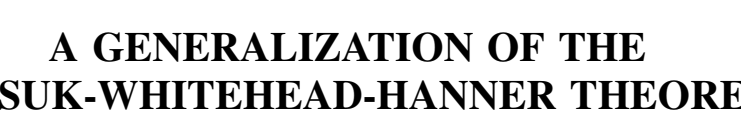




\title{
A GENERALIZATION OF THE BORSUK- WHITEHEAD-HANNER THEOREM
}

\author{
D. M. HYMAN
}

Let $A$ and $B$ be metric spaces and let $f: A \rightarrow B$ be a map. Suppose that $X$ and $Y$ are ANR's containing $A$ and $B$, respectively, as closed subsets, and consider $f$ to be a map from $A$ into $Y$. One of the results of this paper is that the question as to whether or not the adjunction space $X \bigcup_{f} Y$ is an absolute neighborhood extensor for metric pairs (or ANR if $X \bigcup_{f} Y$ is metrizable) depends only on $f$ and not on $X$ and $Y$; that is, if $X \bigcup_{f} Y$ is an ANE (metric) and if $X$ and $Y$ are replaced by ANR's $X^{\prime}$ and $Y^{\prime}$, respectively, then $X^{\prime} \bigcup_{f} Y^{\prime}$ is an ANE (metric). This result is a consequence of the main theorem: Let $B$ be a strong neighborhood deformation retract of a space $Y$ and suppose that both $B$ and $Y-B$ are ANE (metric). If $Y-B$ has a certain type of covering, then $Y$ is an ANE (metric). This generalizes the known result that if $Y$ is metrizable, then $Y$ is an ANR.

By a pair $(X, A)$ we shall mean a space $X$ together with a closed subset $A$. If a space $Y$ has the property that for every metric pair $(X, A)$, each map $f: A \rightarrow Y$ has a neighborhood extension, then $Y$ is called an absolute neighborhood extensor for metric pairs (abbreviated ANE). In particular, a space is an ANR if and only if it is a metrizable ANE [2].

Let $(X, A)$ be a pair, and let $f: A \rightarrow Y$ be a map. It is well known [4, p. 178] that if $X, A$ and $Y$ are ANR's, then the adjunction space $X \mathrm{U}_{f} Y$ is an ANR provided that it is metrizable. This result was essentially proved in successive stages by Borsuk [1], Whitehead [7], and Hanner [3]. Our purpose is to generalize this theorem.

The author wishes to thank Professors James Dugundji and A. H. Kruse for many valuable suggestions.

2. The main theorem. Let $(Y, B)$ be a pair. Generalizing the notion of a canonical cover [2], we say that a collection $\left\{V_{\alpha}\right\}$ of open subsets of $Y$ is a semi-canonical cover of $(Y, B)$ if (1) $\mathrm{U}_{\alpha} V_{\alpha}=Y-B$ and (2) for each $b \in B$ and each neighborhood $U$ of $b$ there is a neighborhood $W$ of $b$ such that $V_{\alpha} \subset U$ whenever $V_{\alpha}$ meets $W .{ }^{1}$ If a semi-canonical cover exists for a pair $(Y, B)$, we call $(Y, B)$ a semicanonical pair.

For later use, we establish the following simple property of semi-

1 A semi-canonical cover differs from a canonical cover only in that a semicanonical cover is not required to be locally finite. 
canonical covers.

Lemma 2.1. Suppose that $\left\{V_{\alpha}\right\}$ is a semi-canonical cover for a pair $(Y, B)$. Let $\left\{x_{\nu}\right\}$ and $\left\{y_{\nu}\right\}$ be two nets in $Y-B$, and suppose that for each $\nu, x_{\nu}$ and $y_{\nu}$ lie in a common element $V_{\nu}$ of $\left\{V_{\alpha}\right\}$. Then $\left\{x_{\nu}\right\}$ converges to a point $b \in B$ if and only if $\left\{y_{\nu}\right\}$ converges to $b$.

Proof. Suppose that $\left\{x_{\nu}\right\}$ converges to $b$. Let $U$ be any neighborhood of $b$, and let $W$ be a neighborhood of $b$ such that $V_{\alpha} \subset U$ whenever $V_{\alpha} \cap W \neq \varnothing$. Since $\left\{x_{\nu}\right\}$ is eventually in $W$, the $\operatorname{sets}\left\{V_{\nu}\right\}$ eventually lie in $U$, and since $y_{\nu} \in V_{\nu}$, it follows that $\left\{y_{\nu}\right\}$ converges to $b$. The converse is proved similarly.

REMARK. If $\left\{V_{\alpha}\right\}$ is a semi-canonical cover of $(Y, B)$ and if for each $y \in Y-B$ an element-call it $V_{y}$-of $\left\{V_{\alpha}\right\}$ containing $y$ is chosen, then the collection $\left\{V_{y}\right\}, y \in Y-B$, is a semi-canonical cover of $(Y, B)$.

A closed subset $B \subset Y$ is called a strong neighborhood deformation retract of $Y$ if there exists a neighborhood $W$ of $B$ and a homotopy $h: W \times I \rightarrow Y$ such that $h_{0}$ is the inclusion, $h_{1}$ is a retraction of $W$ onto $B$, and $h(b, t)=b$ for all $b \in B, t \in I$. $h$ is called a strong deformation retraction of $W$ onto $B$.

We now establish the main theorem.

THEOREM 2.2. Let $(Y, B)$ be a semi-canonical pair such that $B$ is a strong neighborhood deformation retract of $Y$. If both $B$ and $Y-B$ are ANE, then $Y$ is an ANE.

Proof. By hypothesis, there exists a strong deformation retraction $h: W \times I \rightarrow Y$ onto $B$. Let $\left\{V_{y}\right\}, y \in Y-B$, be a semi-canonical cover for $(Y, B)$ as in the remark above.

To prove that $Y$ is an ANE it is sufficient to show that for any metric pair $(X, A)$, each map $f: A \rightarrow W$ has a neighborhood extension $F: U \rightarrow Y$. For from this it follows first that $F \mid F^{-1}(W): F^{-1}(W) \rightarrow$ $W$ is a neighborhood extension of $f$, so that $W$ is an ANE; and then $Y$, being the union of the open ANE subspaces $W$ and $Y-B$, is itself an ANE [4, p. 44]. Given $(X, A)$ and $f: A \rightarrow W$, we proceed to construct $F$.

Let $A_{0}=f^{-1}(B), A_{1}=A-A_{0}$ and $X_{1}=X-A_{0}$. Then $f\left(A_{1}\right) \subset Y-B$, and since $Y-B$ is an ANE, there is a neighborhood $G_{1}$ of $A_{1}$ in $X_{1}$ and a map $\dot{\phi}_{1}: G_{1} \rightarrow Y-B$ such that $\phi_{1}\left|A_{1}=f\right| A_{1}$. Let $d$ be a metric on $X$. For each $a \in A_{1}$, let $G_{a}$ be the set of points $x$ in $G_{1}$ such that

(1) $d\left(x, A_{0}\right)>1 / 2 d\left(a, A_{0}\right)$,

(2) $d(x, a)<d\left(a, A_{0}\right)$, 
(3) $x \in \phi_{1}^{-1}\left(V_{\phi_{1}(a)}\right)$, and

(4) $x \in \phi_{1}^{-1}(W)$.

Let $G_{2}=\bigcup\left\{G_{a} \mid a \in A_{1}\right\} . \quad G_{2}$ is open in $X_{1}$ and contains $A_{1}$. Let $G$ be a neighborhood of $A_{1}$ in $X_{1}$ such that its closure $K$ (in $X_{1}$ ) is contained in $G_{2}$, and let $\lambda: X_{1} \rightarrow[0,1]$ be a map such that $\lambda\left(A_{1}\right)=0$ and $\lambda\left(X_{1}-G\right)=1$. Define $\phi_{2}: K \cup A_{0} \rightarrow Y$ by

$$
\begin{aligned}
\phi_{2}(x) & =h\left(\phi_{1}(x), \lambda(x)\right) & & \text { if } x \in K, \\
& =f(x) & & \text { if } x \in A_{0} .
\end{aligned}
$$

$\phi_{2}$ is well-defined and extends $f$. Furthermore, $\phi_{2}$ is clearly continuous except possibly at those points of $A_{0}$ which are limit points of $K-A_{1}$. To prove its continuity at these points also, we suppose $a \in A_{0}$ is the limit of a sequence $\left\{x_{n}\right\}$ in $K-A_{1}$ and show that $\left\{\dot{\phi}_{2}\left(x_{n}\right)\right\}$ converges to $\phi_{2}(a)$. For each $n$, choose $a_{n} \in A_{1}$ such that $x_{n} \in G_{a_{n}}$. Since $\left\{x_{n}\right\}$ converges to $a \in A_{0}$, it follows from (1) that $\left\{d\left(a_{n}, A_{0}\right)\right\} \rightarrow 0$, and from (2) that $d\left\{\left(x_{n}, a_{n}\right)\right\} \rightarrow 0$. Therefore $\left\{a_{n}\right\}$ converges to $a$. Since $\left\{\phi_{1}\left(a_{n}\right)\right\}=$ $\left\{f\left(a_{n}\right)\right\}$ converges to $f(a)$, we find by $(3)$ and 2.1 that $\left\{\dot{\phi}_{1}\left(x_{n}\right)\right\}$ converges to $f(a)$. Given a neighborhood $V$ of $f(a)$ in $Y$, there is a neighborhood $V_{1}$ of $f(\alpha)$ such that $h\left(V_{1} \times I\right) \subset V$. Since $\left\{\phi_{1}\left(2_{n}\right)\right\}$ converges to $f(\alpha)$, $\left\{\phi_{1}\left(x_{n}\right)\right\}$ is eventually in $V_{1}$, and by the definition of $\dot{\phi}_{2},\left\{\phi_{2}\left(x_{n}\right)\right\}$ is eventually in $V$. Therefore $\phi_{2}$ is continuous at $a$, and hence is continuous on $K \cup A_{0}$.

Since $\lambda=1$ on the boundary (in $X_{1}$ ) of $G$, and since $h$ maps $W \times 1$ into $B$, it follows that $\dot{\phi}_{2}$ maps the boundary (in $X$ ) of $K \cup A_{0}$ into $B$. Since $B$ is an ANE, it follows that $\phi_{2}$ has an extension $F: U \rightarrow Y$ for some open set $U$ in $X$, and the proof is complete.

3. Applications. In order to apply Theorem 2.2, it is necessary to have on hand some semi-canonical pairs. For this purpose we establish.

LEMMA 3.1. Every metric pair $(Y, B)$ is semi-canonical.

Proof. As in [2], for each $y \in Y-B$ let $V_{y}$ be the open $\varepsilon / 2$ ball centered at $y$, where $\varepsilon$ is the distance from $y$ to $B$ under some fixed metric for $Y$. The collection $\left\{V_{y}\right\}$ is a semi-canonical cover for $(Y, B)$.

Combining 3.1 and 2.2, we obtain the following result, which was first proved in [5]:

THEOREM 3.2. (Kruse-Liebnitz). Let $(Y, B)$ be a metric pair such that $B$ is a strong neighborhood deformation retract of $Y$. If $B$ and $Y-B$ are ANR's, then $Y$ is an ANR.

Given a metric space $A$, let $\operatorname{ANR}(A)$ denote the class of all ANR's 
that contain $A$ as a closed subset. Let $f$ be a map from $A$ into an ANR $Y$. Our next result (3.5) states that either the adjunction space $X \cup_{f} Y$ is an $\operatorname{ANE}$ for every $X \in \operatorname{ANR}(A)$ or for no $X \in \operatorname{ANR}(A)$. Therefore, given an $X \in \operatorname{ANR}(A)$, the question of whether or not $X \mathrm{U}_{f} Y$ is an ANE depends only on the map $f$, and not on the choice of $X$.

To obtain this result from 2.2, some additional information concerning semi-canonical covers and strong neighborhood deformation retractions will be needed. The necessary facts are supplied by the following lemmas.

For any pair $(X, A)$ and map $f: A \rightarrow Y$, let $X+Y$ denote the disjoint union of $X$ and $Y$, and let $p: X+Y \rightarrow X \mathbf{U}_{f} Y$ be the natural projection.

Lemma 3.3. Let $(X, A)$ be a pair and let $f: A \rightarrow Y$ be a map. If $\left\{V_{\alpha}\right\}$ is a semi-canonical cover for $(X+Y, A+Y)$, then $\left\{p\left(V_{\alpha}\right)\right\}$ is a semi-canonical cover for $\left(X \mathbf{U}_{f} Y, p(Y)\right)$.

Proof. Since $p$ maps $X-A$ homeomorphically onto $X \cup_{f} Y-$ $p(Y)$, it follows that each $p\left(V_{\alpha}\right)$ is open and $\mathbf{U}_{\alpha} p\left(V_{\alpha}\right)=X \mathbf{U}_{f} Y-$ $p(Y)$. Let $y \in p(Y)$ and let $U$ be a neighborhood of $y$. Since $\left\{V_{\alpha}\right\}$ is semi-canonical, for each $x \in p^{-1}(U \cap p(Y))$ there is a neighborhood $W_{x} \subset p^{-1}(U)$ such that $V_{\alpha} \subset p^{-1}(U)$ whenever $V_{\alpha} \cap W_{x} \neq \varnothing$. Let $W=\bigcup\left\{W_{x} \mid x \in p^{-1}(U \cap p(Y))\right\}$.

From our construction it is clear that $y \in p(W)$ and that $p\left(V_{\alpha}\right) \subset U$ whenever $p\left(V_{\alpha}\right) \cap p(W) \neq \varnothing$. It remains to show that $p(W)$ is open. Since $p$ is an identification, it is sufficient to show that $W$ is saturated, that is, $W=p^{-1}(S)$ for some $S \subset X \bigcup_{f} Y$. From our construction we have $W \cap p^{-1}(p(Y))=p^{-1}(U) \cap p^{-1}(p(Y))=p^{-1}(U \cap p(Y))$. Moreover, since $p$ is one-to-one on $(X+Y)-p^{-1}(p(Y))$ it follows that $W-$ $p^{-1}(p(Y))$ is saturated. Since $W$ is the union of the saturated sets $W \cap p^{-1}(p(Y))$ and $W-p^{-1}(p(Y)), W$ itself is saturated, and the lemma is proved.

Lemma 3.4. Let $X$ and $Y$ be ANR's, and let $f: A \rightarrow Y$ be a map, where $A$ is a closed subset of $X$. Then $X \mathbf{U}_{f} Y$ is an ANE if and only if $p(Y)$ is a strong neighborhood deformation retract of $X \mathbf{U}_{f} Y$.

Proof. Suppose that $X \mathrm{U}_{f} Y$ is an ANE. Since $Y$ is an ANR, $f$ has an extension $F: \bar{U} \rightarrow Y$, where $U$ is some neighborhood of $A$ in $X$. Define a map $g: X \times\{0\} \cup A \times I \cup \bar{U} \times\{1\} \rightarrow X \cup_{f} Y$ by 


$$
\begin{array}{ll}
g(x, 0)=p(x) & \text { if } x \in X ; \\
g(a, t)=p(a) & \text { if } a \in A, \quad 0 \leqq t \leqq 1 ; \\
g(x, 1)=p F(x) & \text { if } x \in \bar{U} .
\end{array}
$$

Since $X \cup_{f} Y$ is an ANE, $g$ has an extension $G: V \rightarrow X \bigcup_{f} Y$, for some open subset $V$ of $X \times I$. Let $W$ be a neighborhood of $A$ in $X$ such that $W \times I \subset V$. The map $h: p(W+Y) \times I \rightarrow X \cup_{f} Y$ defined by

$$
\begin{aligned}
& h(z, t)=G\left((p \mid X)^{-1}(z), t\right) \quad \text { if } z \in p(W), \quad 0 \leqq t \leqq 1, \\
& =z \quad \text { if } z \in p(Y), \quad 0 \leqq t \leqq 1 \text {, }
\end{aligned}
$$

is the desired deformation.

The converse is an immediate consequence of 3.3 and 2.2.

We now obtain the main result of this section.

THEOREM 3.5. Let $f$ be a map from an arbitrary metric space $A$ into an ANR $Y$. If $X_{0} \bigcup_{f} Y$ is an ANE for some $X_{0} \in \operatorname{ANR}(A)$, then $X \mathrm{U}_{f} Y$ is an $\operatorname{ANE}$ for every $X \in \operatorname{ANR}(A)$.

Proof. Given $X \in \operatorname{ANR}(A)$, let $p: X+Y \rightarrow X \bigcup_{f} Y$ and $q: X_{0}+Y \rightarrow$ $X_{0} \cup_{f} Y$ be the natural projections. To prove that $X \cup_{f} Y$ is an ANE it is sufficient, by 3.4 , to show that $p(Y)$ is a strong neighborhood deformation retract of $X \mathrm{U}_{f} Y$.

Since $X$ is an ANR, there exists a neighborhood $G$ of $A$ in $X_{0}$ and a map $\phi: G \rightarrow X$ such that $\phi \mid A$ is the identity map. By 3.4, there is a neighborhood $W$ of $q(Y)$ in $X_{0} \mathbf{U}_{f} Y$ and a strong deformation retraction $h$ of $W$ onto $q(Y)$ over $q(G+Y)$. Since $q^{-1}(W) \cap X_{0}$ is open in $X_{0}, q^{-1}(W) \cap X_{0}$ is an ANR; therefore there exists a neighborhood $U$ of $A$ in $X$ and a map $\psi: U \rightarrow q^{-1}(W) \cap X_{0}$ such that $\psi \mid A$ is the identity map. Since $U$ is open in $X, U$ is an ANR; and it follows that there exists a neighborhood $V$ of $A$ in $U$ and a deformation $j: V \times I \rightarrow U$ such that $j(a, t)=a$, for all $a \in A, 0 \leqq t \leqq 1$, and such that $j_{1}=\phi \psi \mid V$. Letting $\phi+1_{Y}: G+Y \rightarrow X+Y$ be the map defined by $\phi$ and the identity on $Y$, define a map $k: p(V+Y) \times I \rightarrow$ $X \cup_{f} Y$ by

$$
\begin{aligned}
k_{t}(z) & =p j_{2 t}(p \mid X)^{-1}(z) & & \text { if } z \in p(V), 0 \leqq t \leqq 1 / 2, \\
& =p\left(\phi+1_{Y}\right) q^{-1} h_{2 t-1} q \psi(p \mid X)^{-1}(z) & & \text { if } z \in p(V), 1 / 2 \leqq t \leqq 1, \\
& =z & & \text { if } z \in p(Y), 0 \leqq t \leqq 1 .
\end{aligned}
$$

It is easily verified that $k$ is a strong deformation retraction of $p(V+Y)$ onto $p(Y)$, and the proof is complete.

An application of 3.5 gives a direct generalization of the $\mathrm{BWH}$ theorem: 
Corollary 3.6. Let $(X, A)$ be a pair, and let $f: A \rightarrow Y$ be a map. If $X, A$ and $Y$ are ANR's, then $X \mathbf{U}_{f} Y$ is an ANE.

Proof. This result can be obtained as a consequence of 3.3 and 2.2, but it also follows quite simply from 3.5: Taking $X_{0}=A$, we see that $X_{0} \mathrm{U}_{f} Y$ is an ANR, since it is homeomorphic to $Y$. Therefore by $3.5, X \cup_{f} Y$ is an ANE.

If we take $Y$ in 3.5 to be a single point, we obtain

Corollary 3.7. If $A$ is a metric space, then either $X / A$ is an $\operatorname{ANE}$ for every $X \in \operatorname{ANR}(A)$ or for no $X \in \operatorname{ANR}(A)$.

If $A$ is a compact subset of a metric space $X$, then $X / A$ is metrizable [6]. Therefore we have from 3.7

CoROllary 3.8. If $A$ is a compact metric space, then either $X / A$ is an $\operatorname{ANR}$ for every $X \in \operatorname{ANR}(A)$ or for no $X \in \operatorname{ANR}(A)$.

We have seen that for a map $f: A \rightarrow Y$, the question of whether or not $X \cup_{f} Y$ is an ANE is independent of the choice of $X \in \operatorname{ANR}(A)$. Our final result, which slightly generalizes 3.5 , shows that this question is also independent of $Y$. Precisely, we have

Theorem 3.9. Let $A$ and $B$ be metric spaces and let $f: A \rightarrow B$ be a map. Either $X \bigcup_{f} Y$ is an $\mathrm{ANE}$ for every $X \in \operatorname{ANR}(A)$ and $Y \in \operatorname{ANR}(B)$ or for no $X \in \operatorname{ANR}(A)$ and $Y \in \operatorname{ANR}(B)$.

REMARK. For $Y \in \operatorname{ANR}(B)$, we consider $f$ to be not only a map from $A$ into $B$ but also from $A$ into $Y$. This justifies the symbol $X \mathbf{U}_{f} Y$.

Proof of Theorem. Suppose that $X \cup_{f} Y_{0}$ is an ANE for some $X \in \operatorname{ANR}(A)$ and some $Y_{0} \in \operatorname{ANR}(B)$. In view of 3.5 , we need only to show that if $Y \in \operatorname{ANR}(B)$ then $X \mathrm{U}_{f} Y$ is an ANE.

Since $Y$ is an ANR, there is a neighborhood $U$ of $B$ in $Y_{0}$ and a map $\phi: U \rightarrow Y$ such that $\phi(b)=b$ for all $b \in B$.

Letting $\quad p: X+Y \rightarrow X \cup_{f} Y$ and $q: X+U \rightarrow X \cup_{f} U$ be the natural projections, define a map $\psi: X \cup_{f} U \rightarrow X \cup_{f} Y$ by

$$
\begin{aligned}
\psi(z) & =p(q \mid X)^{-1}(z) & & \text { if } z \in q(X), \\
& =p \dot{\phi}(q \mid U)^{-1}(z) & & \text { if } z \in q(U) .
\end{aligned}
$$

$X \mathbf{U}_{f} U$ is open in $X \cup_{f} Y_{0}$, and therefore $X \cup_{f} U$ is an ANE. By 3.4 there is a strong deformation retraction $h$ of an open set $W$ onto $q(U)$ in $X \cup_{f} U$. Define a homotopy $k_{t}: \psi(W) \cup p(Y) \rightarrow X \cup_{f} Y$ by 


$$
\begin{aligned}
k_{t}(z) & =\psi h_{t} \psi^{-1}(z) & & \text { if } z \in \psi(W), \\
& =z & & \text { if } z \in p(Y) .
\end{aligned}
$$

It follows from the equation $\psi(W) \cup p(Y)=p\left((q \mid X)^{-1}(W)+Y\right)$ that $\psi(W) \cup p(Y)$ is an open subset of $X \mathrm{U}_{f} Y$, and it is easily verified that $k$ is a strong deformation retraction of $\psi(W) \cup p(Y)$ onto $p(Y)$. The result now follows from 3.4.

4. Results for AR's. In this section we establish results for AR's and AE's analogous to Theorems 2.2 and 3.9. A space $Y$ is called an absolute extensor for metric pairs (abbreviated $\mathrm{AE}$ ) if for every metric pair $(X, A)$ each map $f: A \rightarrow Y$ has an extension $F: X \rightarrow Y$. A link between AE's and ANE's is provided by the following

LEMma 4.1. If $Y$ is an ANE and if $Y$ can be deformed into an $\mathrm{AE}$ subspace, then $Y$ is an $\mathrm{AE}$.

Proof. Let $B \subset Y$ be an $\mathrm{AE}$ and let $h: Y \times I \rightarrow Y$ be a deformation such that $h_{1}(Y) \subset B$. Suppose that $(X, A)$ is a metric pair and let $f: A \rightarrow Y$ be a map. Since $Y$ is an ANE, there is a neighborhood $U$ of $A$ in $X$ and an extension $F: \bar{U} \rightarrow Y$ of $f$. Let $g: X \rightarrow[0,1]$ be a map such that $g(A)=0$ and $g(X-U)=1$. Since $B$ is an $\mathrm{AE}$, there is a map $G: X-U \rightarrow B$ such that $G \mid$ bdry $U=h_{1} F \mid$ bdry $U$. Define a map $\phi: X \rightarrow Y$ by

$$
\begin{aligned}
\phi(x) & =h(F(x), g(x)) & & \text { if } x \in \bar{U}, \\
& =G(x) & & \text { if } x \in X-U .
\end{aligned}
$$

$\phi$ extends $f$, and the lemma is proved.

We now establish the analog of 2.2 .

Theorem 4.2. Let $(Y, B)$ be a semi-canonical pair such that $B$ is a strong deformation retract of $Y$. If $B$ is an $\mathrm{AE}$ and if $Y-B$ is an $\mathrm{ANE}$, then $Y$ is an $\mathrm{AE}$.

Proof. By 2.2, $Y$ is an ANE. Since by hypothesis $Y$ is deformable into $B, Y$ is an $\mathrm{AE}$ by 4.1 .

In order to obtain the analog of 3.9 , we will need the analog of 3.4 .

Lemma 4.3. Let $X$ and $Y$ be AR's, and let $f: A \rightarrow Y$ be a map, where $A$ is a closed subset of $X$. Then $X \cup_{f} Y$ is an $\mathrm{AE}$ if and only if $p(Y)$ is a strong deformation retract of $X \mathbf{U}_{f} Y$. 
Proof. Suppose that $X \mathrm{U}_{f} Y$ is an AE. Since $Y$ is an AR, $f$ has an extension $F: X \rightarrow Y$. Since $X \cup_{f} Y$ is an AE, the map

$$
g: X \times\{0\} \cup A \times I \cup X \times\{1\} \rightarrow X \bigcup_{f} Y
$$

defined by

$$
\begin{array}{ll}
g(x, 0)=p(x) & \text { if } x \in X, \\
g(a, t)=p(a) & \text { if } a \in A, \quad 0 \leqq t \leqq 1, \\
g(x, 1)=p F(x) & \text { if } x \in X,
\end{array}
$$

has an extension $G: X \times I \rightarrow X \bigcup_{f} Y$. The map $h: X \bigcup_{f} Y \times I \rightarrow$ $X \cup_{f} Y$ defined by

$$
\begin{aligned}
& h(z, t)=G\left((p \mid X)^{-1}(z), t\right) \quad \text { if } z \in p(X), \quad 0 \leqq t \leqq 1 \\
& =z \quad \text { if } z \in p(Y), \quad 0 \leqq t \leqq 1
\end{aligned}
$$

is the desired deformation.

Conversely, if $p(Y)$ is a strong deformation retract of $X \mathbf{U}_{f} Y$, then $X \cup_{f} Y$ is an ANE by 3.4 and an $\mathrm{AE}$ by 4.1 .

We now establish the analog of 3.9 .

Theorem 4.4. Let $A$ and $B$ be metric spaces and let $f: A \rightarrow B$ be a map. Either $X \cup_{f} Y$ is an $\mathrm{AE}$ for every $X \in \mathrm{AR}(A)$ and $Y \in \operatorname{AR}(B)$ or for no $X \in \operatorname{AR}(A)$ and $Y \in \operatorname{AR}(B)$.

Proof. Suppose $X_{0} \bigcup_{f} Y_{0}$ is an $\mathrm{AE}$ for some $X_{0} \in \mathrm{AR}(A)$ and $Y_{0} \in \mathrm{AR}(B)$, and suppose $X \in \mathrm{AR}(A)$ and $Y \in \mathrm{AR}(B)$. Let $p: X+Y \rightarrow$ $X \mathbf{U}_{f} Y$ and $q: X_{0}+Y_{0} \rightarrow X_{0} \mathbf{U}_{f} Y_{0}$ be the natural projections.

By 3.9, $X \mathrm{U}_{f} Y$ is an $\mathrm{ANE}$; to prove that it is an $\mathrm{AE}$ it is sufficient, by 4.3 , to show that $X \mathbf{U}_{f} Y$ can be deformed into $p(Y)$. Since $X$ and $X_{0}$ are AR's, there are maps $\phi: X \rightarrow X_{0}$ and $\phi_{0}: X_{0} \rightarrow X$, each extending the identity on $A$, and a deformation $j_{t}$ on $X$ leaving $A$ pointwise fixed and such that $j_{1}=\phi_{0} \phi$. Similarly, there are maps $\psi: Y \rightarrow Y_{0}$ and $\psi_{0}: Y_{0} \rightarrow Y$, each extending the identity on $B$, and a deformation $k_{t}$ on $Y$ leaving $B$ pointwise fixed and such that $k_{1}=\psi_{0} \psi$. By 4.3, there is a strong deformation retraction $h_{t}$ of $X_{0} \cup_{f} Y_{0}$ onto $q\left(Y_{0}\right)$. Define a deformation $g_{t}$ on $X \mathbf{U}_{f} Y$ by

$$
\begin{aligned}
g_{t}(z) & =p j_{2 t}(p \mid X)^{-1}(z) & & \text { if } z \in p(X), 0 \leqq t \leqq 1 / 2, \\
& =p k_{2 t}(p \mid Y)^{-1}(z) & & \text { if } z \in p(Y), \quad 0 \leqq t \leqq 1 / 2, \\
& =p\left(\dot{\phi}_{0}+\psi_{0}\right) q^{-1} h_{2 t-1} q \phi(p \mid X)^{-1}(z) & & \text { if } z \in p(X), \quad 1 / 2 \leqq t \leqq 1, \\
& =p\left(\dot{\phi}_{0}+\dot{\psi}_{0}\right) q^{-1} h_{2 t-1} q \psi(p \mid Y)^{-1}(z) & & \text { if } z \in p(Y), \quad 1 / 2 \leqq t \leqq 1,
\end{aligned}
$$

where $\phi_{0}+\psi_{0}: X_{0}+Y_{0} \rightarrow X+Y$ is the map defined by $\phi_{0}$ and $\psi_{0}$. $g$ 
deforms $X \mathbf{U}_{f} Y$ into $p(Y)$, and the proof is complete.

By taking $B$ to be a single point, we obtain

Corollary 4.5. If $A$ is a metric space, then either $X / A$ is an $\mathrm{AE}$ for every $X \in \mathrm{AR}(A)$ or for no $X \in \mathrm{AR}(A)$.

Corollary 4.6. If $A$ is a compact metric space, then either $X / A$ is an $\mathrm{AR}$ for every $X \in \mathrm{AR}(A)$ or for no $X \in \mathrm{AR}(A)$.

\section{REFERENCES}

1. K. Borsuk, Uber eine Klasse von lokal zusammenhangenden Raumen, Fundam. Math. 19 (1932), 220-242.

2. J. Dugundji, An extension of Tietze's theorem, Pacific J. Math. 1 (1951), 353-367.

3. O. Hanner, Some theorems on absolute neighborhood retracts, Arkiv. Math. 1 (1951), 389-408.

4. S. T. Hu, Theory of Retracts, Wayne State Press, Detroit, 1965.

5. A. H. Kruse and P. W. Liebnitz, An application of a family homotopy extension theorem to ANR spaces, Pacific J. Math. 16 (1966), 331-336.

6. A. H. Stone, Metrizability of decomposition spaces, Proc. Amer. Math. Soc. 7 (1956), 690-700.

7. J. H. C. Whitehead, Note on a theorem due to Borsuk, Bull. Amer. Math. Soc. 54 (1948), 1125-1132.

Received December 12, 1966.

UNIVERSity OF SOUTherN CALIForNIA 



\section{PACIFIC JOURNAL OF MATHEMATICS}

\section{EDITORS}

\section{H. ROYDEN \\ Stanford University \\ Stanford, California}

\author{
J. P. JANS \\ University of Washington \\ Seattle, Washington 98105
}

J. DugundJI

Department of Mathematics

Rice University

Houston, Texas 77001

RichaRd ARENS

University of California

Los Angeles, California 90024

\section{ASSOCIATE EDITORS}
E. F. BECKENBACH
B. H. NeumanN
F. WOLF
K. YOSIDA

\section{SUPPORTING INSTITUTIONS}

\author{
UNIVERSITY OF BRITISH COLUMBIA \\ CALIFORNIA INSTITUTE OF TECHNOLOGY \\ UNIVERSITY OF CALIFORNIA \\ MONTANA STATE UNIVERSITY \\ UNIVERSITY OF NEVADA \\ NEW MEXICO STATE UNIVERSITY \\ OREGON STATE UNIVERSITY \\ UNIVERSITY OF OREGON \\ OSAKA UNIVERSITY \\ UNIVERSITY OF SOUTHERN CALIFORNIA
}

\author{
STANFORD UNIVERSITY \\ UNIVERSITY OF TOKYO \\ UNIVERSITY OF UTAH \\ WASHINGTON STATE UNIVERSITY \\ UNIVERSITY OF WASHINGTON

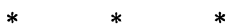 \\ AMERICAN MATHEMATICAL SOCIETY \\ CHEVRON RESEARCH CORPORATION \\ TRW SYSTEMS \\ NAVAL ORDNANCE TEST STATION
}

Mathematical papers intended for publication in the Pacific Journal of Mathematics should be typewritten (double spaced). The first paragraph or two must be capable of being used separately as a synopsis of the entire paper. It should not contain references to the bibliography. Manuscripts may be sent to any one of the four editors. All other communications to the editors should be addressed to the managing editor, Richard Arens at the University of California, Los Angeles, California 90024.

50 reprints per author of each article are furnished free of charge; additional copies may be obtained at cost in multiples of 50 .

The Pacific Journal of Mathematics is published monthly. Effective with Volume 16 the price per volume (3 numbers) is $\$ 8.00$; single issues, $\$ 3.00$. Special price for current issues to individual faculty members of supporting institutions and to individual members of the American Mathematical Society: $\$ 4.00$ per volume; single issues $\$ 1.50$. Back numbers are available.

Subscriptions, orders for back numbers, and changes of address should be sent to Pacific Journal of Mathematics, 103 Highland Boulevard, Berkeley 8, California.

Printed at Kokusai Bunken Insatsusha (International Academic Printing Co., Ltd.), 7-17, Fujimi 2-chome, Chiyoda-ku, Tokyo, Japan.

PUBLISHED BY PACIFIC JOURNAL OF MATHEMATICS, A NON-PROFIT CORPORATION

The Supporting Institutions listed above contribute to the cost of publication of this Journal, but they are not owners or publishers and have no responsibility for its content or policies. 


\section{Pacific Journal of Mathematics}

\section{Vol. 23, No. 2 \\ April, 1967}

Herbert Stanley Bear, Jr. and Bertram John Walsh, Integral kernel for

one-part function spaces .......................... 209

Mario Borelli, Some results on ampleness and divisorial schemes ....... 217

John A. Erdos, Unitary invariants for nests . ................... 229

Nathaniel Grossman, The volume of a totally-geodesic hypersurface in a pinched manifold.................................. 257

D. M. Hyman, A generalization of the Borsuk-Whitehead-Hanner theorem ............................................. 263

I. Martin (Irving) Isaacs, Finite groups with small character degrees and large prime divisors ............................. 273

I. Martin (Irving) Isaacs, Two solvability theorems ................ 281

William Lee Johnson, The characteristic function of a harmonic function in a locally Euclidean space ............................... 291

Ralph David Kopperman, Application of infinitary languages to metric spaces ............................................. 299

John Lauchlin MacDonald, Relative functor representability ............ 311

Mahendra Ganpatrao Nadkarni, A class of measures on the Bohr group.... 321

Keith Lowell Phillips, Hilbert transforms for the p-adic and p-series fields....................................... 329

Norman R. Reilly and Herman Edward Scheiblich, Congruences on regular semigroups ...

Neil William Rickert, Measures whose range is a ball ... . .

Gideon Schwarz, Variations on vector measures

Ronald Cameron Riddell, Spectral concentration for self-adjoint operators. .

Haskell Paul Rosenthal, A characterization of restrictions of

Fourier-Stieltjes transforms ................... 